\title{
The Study of Customer Segmentation Examined by Catastrophe Model
}

\author{
Yu-Kai Huang \\ Institute of Publishing and Culture Enterprise Management \\ osilo.huang@gmail.com
}

\begin{abstract}
Convenience stores in Taiwan have made remarkable successes with retail delivery services by integrating E-commerce and logistics systems to form a new retail delivery model: "On-line shopping with pick-ups at convenience stores." Although choice behavior has been discussed in marketing, few studies describe the non-linear characteristic of choice behavior. The catastrophe model was used to analyze the linkages between customer satisfaction and switching cost on pick-up point service loyalty. The results indicated that the switching cost plays the splitting factor in the catastrophe model, and a high switching cost makes the discontinuous choice behavior. In the cusp catastrophe mode, "Reselection electronic map" is the main index of the splitting factor. Nevertheless, "The quality of relative service concerning the electronic map" is the main index of the normal factor. It has been expected that a catastrophe approach to discontinuous behavior has made clearly abundant implications. Based on the findings of loyalty in the application of cusp catastrophe theory, the cusp catastrophe model is an appropriate model to know the process of loyalty. It suggests that other researchers could consider the cusp catastrophe theory and other nonlinear techniques, especially for standard approaches not adequately to capture the underlying dynamic.
\end{abstract}

\section{Introduction}

The Internet has provided people with a new medium for social activities and has also opened up entirely new features of social reality. In Taiwan, convenience stores,

Please use the following format when citing this chapter:

Huang, Y. -K., 2008, in IFIP International Federation for Information Processing, Volume 286; Towards Sustainable Society on Ubiquitous Networks, eds. Oya, M., Uda, R., Yasunobu, C., (Boston: Springer), pp. $37-48$. 
which are widely distributed, provide a 24-hour purchasing environment for customers. Up to date, the convenience stores have integrated E-commerce with logistics system to form a new retail delivery model: "On-line shopping in an electronic store and pick-up goods in a convenience store." Because convenience stores remain open 24 hours a day even on holidays, on-line shoppers can pick up goods according to their schedules. In Taiwan, portal sites such as Yahoo.com and Pchome.com are providing retail delivery services for on-line customers and have made many remarkable successes.

Taiwan's history of retailing delivery of e-commerce is not long. CVS.com (CVS.com is a joint venture by four families of convenience stores including Family.com, Hi-Life.com, Okcvs.com and Nikomart.com) is an RD provider that began service in the beginning of 2000 in Taiwan, while 7-11.com joined the market at the end of 2000. Because of safe method of payment way and the quick delivery, $\mathrm{RD}$ services by convenience stores have become a substantial provider of the electronic commerce logistics in Taiwan. Currently, over 1,200,000 per month orders are completed by the electronic commerce with retail delivery model. Figure 1 shows the retailing structure.

For a RD (Retailing Delivery) system provider, the business volume of the RD service is mostly based on store locations. Therefore, it is difficult for the provider to provide different services for customers. Generally speaking, 7-11 has the greatest share in the E-commerce retail delivery market. It has captured $70 \%$ of the market while the other convenience stores only account for $30 \%$ of the market. Due to the difficulty for retail delivery providers to make a difference on the operation process, the customer loyalty has become an important issue for marketing practitioners.

The RD system provides an easy on-line shopping process, safe pick-up points and quick delivery service for e-retailing. The retailing delivery includes four functions: (1) e-map, (2) packing process, (3) delivery system and (4) pick-up point. Figure 1 displays the concept of retailing delivery system.

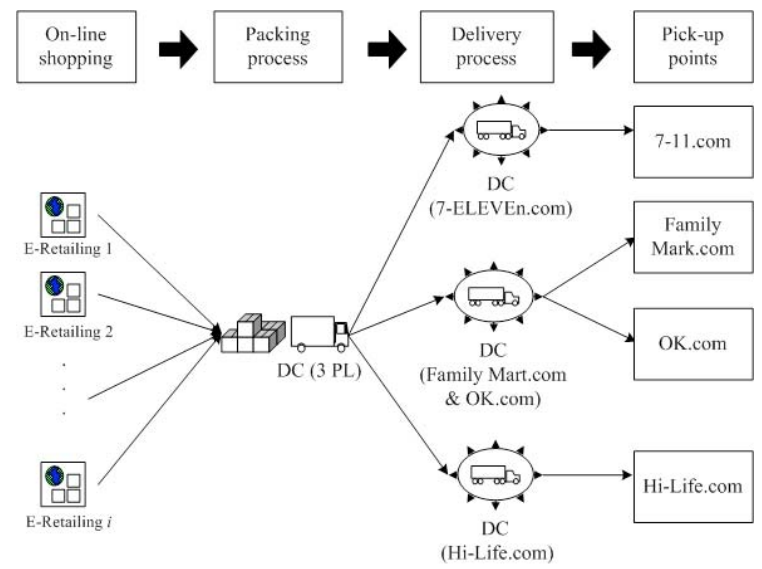

Fig. 1. Concept of retailing delivery system

In the electronic commercial environment, customer preferences are very diverse, 
and their loyalty level is very low. Companies should acknowledge the changes in customer demand patterns quickly and respond to consumer's behavior appropriately. Thus, an important issue is why consumers vary in, how they divide into their purchases across outlets, and how outlets can gain a greater share of consumer expenditures. It is necessary to use management experience and research results to create an overall picture of the relationships between loyalty and satisfaction. The research issues are included in the following:

1. Applying the catastrophe theory and modeling a Catastrophe Cusp Model to the consumer behavior on the pick-up point choice.

2. Using the Cusp Catastrophe Model for developing loyalty strategies.

\section{Theoretical Foundation and Research Method}

\subsection{Cusp Catastrophe Model}

Catastrophe theory is a mathematical theory that describes the relation between two sets of variables, control variables and behavioural variables (state variables), is so-called gradient system. In the gradient system, with fixed value of the control variables, the system always seeks an equilibrium state which means that the value of the behavioural variable changes until the minimum or maximum of a certain quantity is obtained.

Catastrophe theory was developed and popularized in the early 1970's. Initially, it attracted attention very quickly in 1978; an entire issue of behavioral science was devoted to the approach. After a period of criticism, the catastrophe theory is well established and widely applied. Today the theory is very much alive. Numerous nonlinear phenomena that exhibit discontinuous jumps in behavior have been modeled by using the theory, for instance in the field of chemistry [1], physics [2-5], psychology [6] and in the social sciences [7-10]. The models' strengths include that complex behavior can be captured by using significantly fewer nonlinear equations than the number of linear equations needed to describe the same phenomena.

Catastrophes are bifurcations between different equilibria and fixed-point attractors. It is a topological branch of mathematics developed to study and classifies phenomena characterized by sudden shifts in behavior arising from small change in circumstance. The theory specifies that small changes in control parameters across critical thresholds will cause stable equilibria either to disappear, or to bifurcate into multiple equilibria, some of which are stable.

The catastrophe structure most commonly has been applied the cusp model [10]. Figure 2 shows the basic form of the deterministic cusp model generated. Each catastrophe model can be formalized by potential or gradient structures, a potential function $F(x, c)$ is a function of both the system state $x$ and the control parameter(s) $c$. The Cusp Catastrophe Model consists of one behavior variable and only two control variables. The potential is represented by Eq. (1), the equilibria of Eq. (1) is three-dimensional [7]. 


$$
F(u, v, x)=-\frac{1}{4} x^{4}+\frac{1}{2} u x^{2}+v x
$$

Where the state variable $\mathrm{x}$ is controllability, and $u$ and $v$ are environmental control parameters. As a stable equilibrium state $x$ for this potential gives relative value $\mathrm{x}$ of a function $F(u, v, x)$, a set of point $(u, v, x)$ is defined as Eq. (2),

$$
\frac{\partial F}{\partial x}=-x^{3}+u x+v=0 ; M_{F}:\left\{(u, v, x) \mid-x^{3}+u x+v=0\right\}
$$

Where $M_{F}$ is said to be cusp catastrophe manifolded. The values of $x$ in correspondence to which attains a local maximum or minimum satisfies the condition as Eq. (3),

$$
3 x^{2}+u=0
$$

Eliminating $x$ from Eq. (2) and Eq. (3), the bifurcation set is express by Eq. (4).

$$
4 u^{3}=27 v^{2}
$$

A switch in topology takes place at the values of $u$ and $v$ satisfying Eq. (4), which constitute the catastrophe set. In the equation Eq. (3) $x$ is the state variable, and $u, v$ are control parameters. The parameter $u$ determines whether the system has one or can have two stable equilibria. When $u>0$ only one stable equilibrium can exit whatever the value of $v$. When $u<0$ it depends upon the value of $v$ whether the system has a single low level of stable equilibrium, or a low level and a high level equilibria, or a single high level of equilibrium.

According to the different variable sets, three different cases can be defined. Case 1: There is one stable equilibrium point; Case 2: There are two stable and one unstable equilibrium point; or Case 3: There is one stable equilibrium point, and one at which an instantaneous jump in the state variable occurrence.

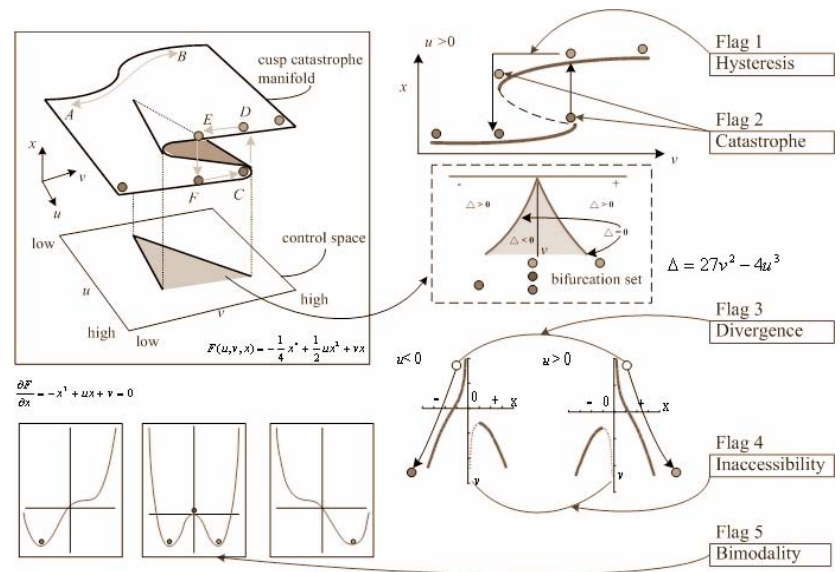

Fig. 2. A Cusp Catastrophe Model and its Five Different Flags

Changes in the control or independent variables ( $v$-right/left movement, and $u$-back/front movement) cause the changes in the behavior or dependent variable ( $x$-vertical movement). If $u$ is low, smooth changes in $v$ occur in proportion to change in $x$ as shown by examining the travel of point $A$ and $B$ in Figure 2. When $u$ is high (past the singularity) changes in $v$ producing relatively small changes in $x$ until a threshold is reached when there is a sudden discontinuous shift in $x$. This is depicted 
by the path from point $C$ to $D$ in Figure 2. Note, that a reversal in $v$ back to the point of the shift in $x$, will not cause $x$ to return back to its original position, since $v$ will have to move well past to cause $x$ to shift back. This is shown as the movement from point $C$ to $E$.

\subsection{Approaches for Estimating Catastrophe Models}

GEMCAT approaches have been successfully applied in a number of different organizational research contexts [7, 11]. Next, the GEMCAT approach is described in some details. Oliva et al.'s [12] GEMCAT approach allows all variables in a catastrophe to be latent composites. To accomplish this, the variable $X, Y$, and $Z$ in the canonical cusp is presented by Equation (5),

$$
f(x, y, z)=\frac{1}{4} z^{4}-\frac{1}{2} y z^{2}-x z
$$

Let:

$i=1 \ldots I$ dependent variables;

$j=1 \ldots J$ "splitting" independent variables;

$k=1 \ldots K$ "normal" independent vatiables;

$t=1 \ldots T$ observations;

$\mathrm{Z}_{i t}=$ the value of the $i$-th dependent variable on observation $t$;

$\mathrm{Y}_{i t}=$ the value of the $j$-th splitting independent variable on observation $t$;

$\mathrm{X}_{k t}=$ the value of the $k$-th normal independent variable on observation $t$;

Now, define three "latent" unobservable variables:

$$
\begin{aligned}
& Z_{t}^{*}=\sum_{i=1}^{I} \alpha_{i} Z_{i t} \\
& Y_{t}^{*}=\sum_{j=1}^{J} \beta_{j} Y_{j t} \\
& X_{t}^{*}=\sum_{k=1}^{K} \gamma_{k} X_{k t}
\end{aligned}
$$

Thus, the equation (5) can be redefined as these three "latent" unobservable constructs which can thus accommodate univariate or multivariate measurements for each type of variable. This allows the cusp catastrophe model to be rewritten as shown in Eq. (9):

$$
f\left(X_{t}^{*}, Y_{t}^{*}, Z_{t}^{*}\right)=\frac{1}{4} Z_{t}^{* 4}-X_{t}^{*} Z_{t}^{*}-\frac{1}{2} Y_{t}^{*} Z_{t}^{* 2}
$$

In these terms, the estimation problem, given $X=X_{k t}, \mathrm{Y}=\mathrm{Y}_{j t}$ and $Z=Z_{i t}$, and its derivative set equal to zero can be stated as:

$$
\frac{\partial f\left(X_{t}^{*}, Y_{t}^{*}, Z_{t}^{*}\right)}{\partial Z_{t}^{*}}=Z_{t}^{*^{3}}-X_{t}^{*}-Y_{t}^{*} Z_{t}^{*}=0
$$

From equation Eq. (10) the estimating goal is to minimize Eq. (11):

$$
\operatorname{Min}_{\alpha i, \beta j, \gamma k} \Phi=\left\|e_{t}^{2}\right\|=\sum_{t=1}^{T}\left[Z_{t}^{*^{3}}-X_{t}^{*}-Y_{t}^{*} Z_{t}^{*}\right]^{2}
$$

where the $e_{t}=$ error. That is, for a given empirical data on various specified dependent, splitting, and normal variables, one wishes to estimate the impact coefficients that define their respective latent variables, which make $\Phi$ as close to 
zero as possible. Minimizing $\Phi$ is equivalent to find the best fitting cusp catastrophe surface to the empirical data.

More recently, Lange et al. (2000) developed an improved version of the algorithm called GEMCAT II (the GEMCAT II software is developed in Delphi V3.0), which provides greater speed, efficiency, utility and flexibility in terms of analysis and testing [13]. GEMCAT II uses a combination of the Downhill Simplex method and Powell's Conjugate Gradient approach. GEMCAT estimates the various indicator weights by minimizing the total squared residual $(\Phi)$ across observations; the default procedure is to run the Downhill Simplex.

\section{Data Analysis and Results}

\subsection{Operationalized of Variable and Data Set}

According to the research by Feng and Huang [14], the two most relevant variables influencing the loyalty of a pick-point are switching cost and service quality. With these two variables, the loyalty dynamics may be conceptualized in terms of the cusp catastrophe model. In our hypothesized model, loyalty is the dependent variable $z$, service quality is a normal variable $v$, whereas the switching cost is a bifurcation variable $u$. Cusp catastrophe theory uses a continuous parameter to describe discrete morphology behavior. In a cusp catastrophe model framework, after the intensities of the normal factor and splitting factor are determined, model fitting and dynamical analyses are then performed.

GEMCAT allows $x, u, v$ in the cusp catastrophe model to represent "latent" variables consisting of arbitrary linear combinations of more elementary "indicator" variable. Our operationalizations of the dependent and independent indicator are measure is as follows:

1. Loyalty $\left(X^{*}\right)$ :

$\triangle x_{1}$ : relative loyalty by repurchase

$\triangle x_{2}$ : relative loyalty by personal partiality

$\triangle x_{3}$ : relative loyalty by verbal communications

$\triangle x_{4}$ : relative loyalty by the partialities of other services

2. Service Quality $\left(V^{*}\right)$

$\triangle v_{l}$ : relative service quality concerning the electronic map

$\triangle v_{2}$ : relative service quality concerning the e-tracking

$\triangle v_{3}$ : relative service quality concerning the service attitude

$\triangle v_{4}$ : relative service quality concerning the marketing program

3. Switching Cost $\left(U^{*}\right)$

$u_{1}$ : switching cost concerning the personal custom

$u_{2}$ : switching cost concerning the electronic map

$u_{3}$ : switching cost concerning the distance 
The data for the study were collected from an on-line survey that a pop-window invitation contained an embedded URL linkage to the website hosting. Participants who completed the questionnaires were given coupons of 100NT through cellular phone announcements. Survey responds were rated on a five-point Likert scale. The survey lasted for six weeks and was collected 11,462 responds in total. A random sample of 1,500 responses generated from those who had experiences of picking up on-line ordered merchandises at convenience stores was selected because of the restriction of GEMCAT $\Pi$. For instance, the latent variables in a cusp catastrophe model take the following general form:

$$
\begin{aligned}
& X_{t}^{*}=\alpha_{1} \Delta x_{1 t}+\alpha_{2} \Delta x_{2 t}+\alpha_{3} \Delta x_{3 t}+\alpha_{4} \Delta x_{4 t}, t=1,2, \ldots, 1500 \\
& V_{t}^{*}=\gamma_{1} \Delta v_{1 t}+\gamma_{2} \Delta v_{2 t}+\gamma_{3} \Delta v_{3 t}+\gamma_{4} \Delta v_{4 t}, t=1,2, \ldots, 1500 \\
& U_{t}^{*}=\beta_{1} u_{1 t}+\beta_{2} u_{2 t}+\beta_{3} u_{3 t}, t=1,2, \ldots, 1500
\end{aligned}
$$

\subsection{Model Fit and Analysis Results}

Data were fitted using GEMCAT II version 1.3, yielding the average squared difference $\Phi / N=0.000929$ between the actual and the predicted $X^{*}$, where $N$ represents the number of cases. Substitution of these weights into Eq. (15)-Eq. (18) yields,

$$
\begin{aligned}
& X^{*}=\Delta x_{1}+0.2813 \cdot \Delta x_{2}+0.2175 \cdot \Delta x_{3}+0.1981 \cdot \Delta x_{4} \\
& U^{*}=0.074 \cdot u_{1}+0.2952 \cdot u_{2}+0.761 \cdot u_{3} \\
& V^{*}=0.0615 \cdot \Delta v_{1}+0.0089 \cdot \Delta v_{2}+0.032 \cdot \Delta v_{3}+0.0021 \cdot \Delta v_{4} \\
& \operatorname{Min} \Phi=\left\|e_{t}^{2}\right\|=\sum_{t=1}^{1500}\left[Z_{t}^{*^{3}}-X_{t}^{*}-Y_{t}^{*} Z_{t}^{*}\right]^{2}=2.871
\end{aligned}
$$

Figure 3 shows the relationships between control variables and dependent variable. Figure 4 explains the distribution situation in the control space of our study samples; the $\mathrm{x}$-axis shows the relative service quality of CVS.com and 7-11.com, while the $y$-axis expresses the switching cost.

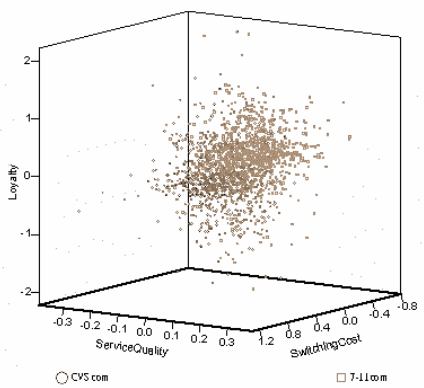

Fig. 3. Relationships between Control Variables and Dependent Variable 


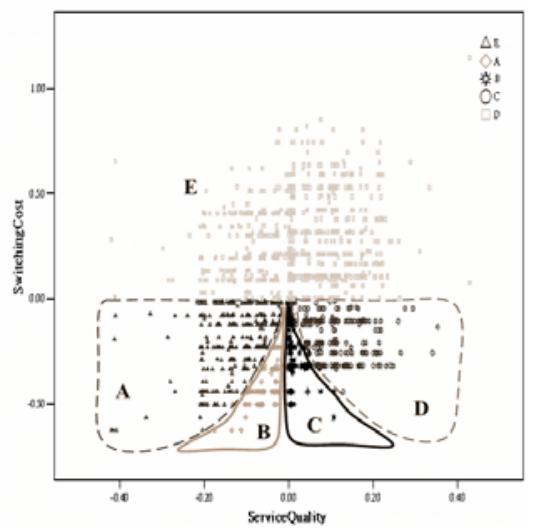

Fig. 4. Control Space Separate from Different Control Set

\section{Discussion and Implications}

In the previous section, we have demonstrated the efficacy of GEMCAT II for testing catastrophe models; next, we will discuss the nonlinear behavior of our behavior model developed by the cusp catastrophe model using software developed of this thesis. Figure 5 presents our mapping of the control variables onto the catastrophe surface structure. The projection of the fold curve into parameter space $C$ yields the catastrophe set $K$. The $b_{4}$ and $b_{3}$ are trajectories in $C$ that determine corresponding trajectories $B_{4}$ and $B_{3}$ of equilibrium behavior.

System dynamics occur on the surface of the model in Figure 5. Changes in position result form changes in $U$ (switching cost) and $V$ (service quality), causing changes in $X$ (loyalty). If $U$ 's magnitude is small, then smooth change occurs in $X$ (loyalty), directly proportional to change in $X$ (loyalty), as depicted by Path $\mathrm{D}$ as shown in Figure 5. Small difference in the initial starting positions (e.g., Points $A_{1}$ and $\mathrm{A}_{2}$ ) can result in vastly different values for $X$ (loyalty) as the magnitude of the $U$ (switching cost) variable increases beyond the point where the pleat starts. This can be seen in Figure 5, where $A_{1}$ is driven downward, and $A_{2}$ is driven upward. At high values of $U$ (switching cost), large changes in $V$ (service quality) will produce relatively small changes in $X$. At some point, however, a sudden discontinuous shift in $X$ (loyalty) will occur that reverses system behavior from its previous state. This is shown by examining the travel from Point $\mathrm{B}_{4}$ to $\mathrm{B}_{3}$ as depicted by Path $\mathrm{C}$ in Figure 5 . Note that once the shift has happened, reversing the values of $V$ will not cause a substantial downward change in $X$ (loyalty). There must be a significant reversal in $U$ (switching cost) before a shift down to point $\mathrm{B}_{4}$ will occur. These lags in response are aggravated or mitigated by the size of the $U$ (switching cost) variable. Within the cusp area, the dependent variable can take on two possible values for a given $(V, U)$ pair. This characteristic allows the modeling of lag effects (hystereses).

Moving from the more tactical issues discussed in the illustration to more strategic issues pertaining to service strategy in general, our research indicates that, depending on switching cost, the relation between service quality and loyalty can be nonlinear, at least for some facets of service. This finding has important implications for loyalty strategies and decisions on how to deploy a limited budget across different 
opportunities to enhance service, given that competitors are making similar decisions. The cusp catastrophe model have five important features, we develop our four different types of loyalty strategies via the catastrophe characteristic and the hysteresis characteristic in this section as shown in Figure 6.

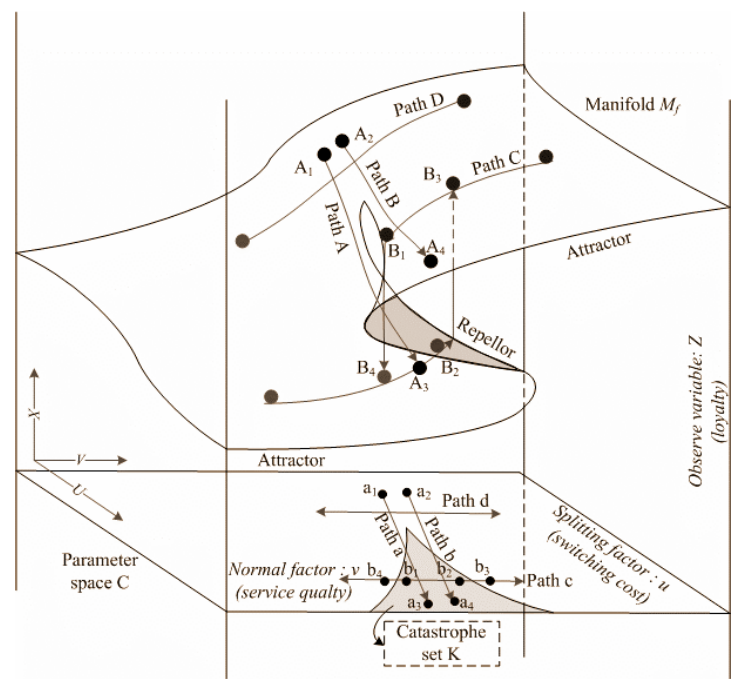

Fig. 5. Behavior Manifold of the Cusp Catastrophe Model

We take CVS.com as an example and develop loyalty strategies via the cusp catastrophe model. Clustering analysis to identify convertible consumers is the first step of developing loyalty strategy. As shown in Figure 6, it is difficult for any strategies to change a consumer if he or she is very satisfied with 7-11.com or switching cost is very high. Idea-convertible consumers must have medium switching cost and there should be difference in service quality between 7-11.com and CVS.com (see Figure 6).

According to our analysis above, switching cost and service quality are the two main factors influencing loyalty. That is, when perceived switching costs are low, dissatisfied consumers should be more likely to defect than satisfied customers because perceived switching benefits outweigh perceived switching costs. Simply speaking, when perceived switching costs are high, customers may remain in spite of their dissatisfaction owing to the perceived switching benefits. Thus, the relationship between customer satisfaction and loyalty is stronger for customers with high switching costs than for those with low switching costs. 


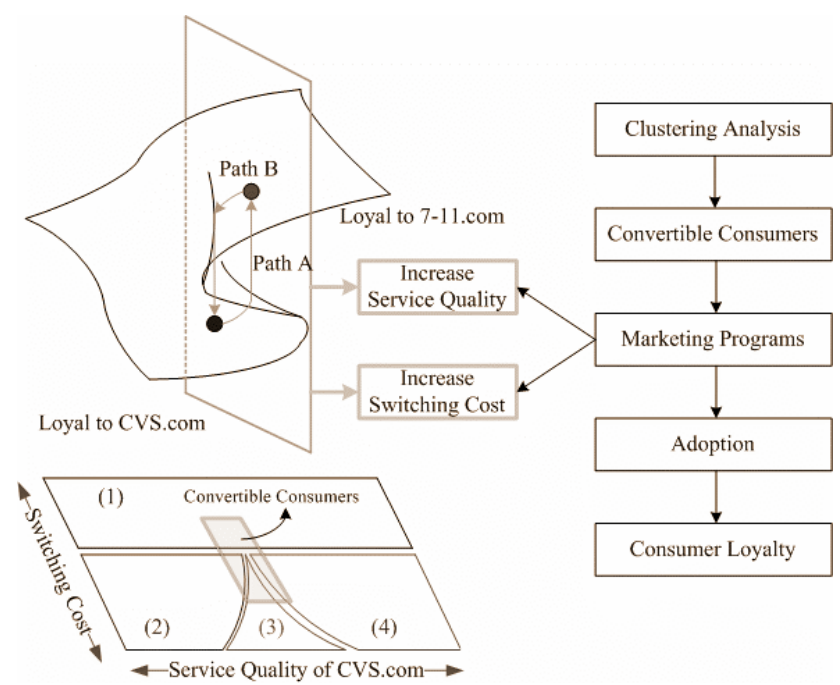

Fig. 6. Developing Loyalty Strategy via Catastrophe Model

Service quality and switching cost are the control variables in our cusp catastrophe model; we will discuss these factors briefly here. Switching cost is the customer's perception of loss in terms of time, money, and psychological costs when switching. Many studies argued that it is defined as perceived risks when switching service providers, the potential losses are of financial, performance-related, social, psychological, and safety-related nature. When a customer changes a service provider, there exists some uncertain perceived risks that a new supplier might not perform the core service at a level equal to, or better than, the current supplier. Clearly, switching costs seem to be an important element when deciding whether to switch a service provider or not. Because the indicator $u_{3}$ (switching cost concerning the distance) has the greatest impact on loyalty among the different indicators of switching cost, one of these loyalty strategies will be related to indicator $u_{3}$.

From the short-term point of view, indicator $v_{4}$ (marketing program) is the most important factor among the four indicators of service quality of the control variables $u_{i}$. Sales promotions can encourage consumers to either buy larger quantities of the promoted product or buy that product at an earlier time. By definition, the objective of sales promotions is to offer a direct incentive that affects consumers to accelerate their purchase.

\section{Conclusions}

Catastrophe Theory (CT) is a theory of great generality that can provide useful insights as to how behavior may radically change as a result of varying smoothly control variables. The theory describes how small, continuous changes in control parameters can have sudden, discontinuous effects on dependent variables. The cusp catastrophe model needs one behavior variable and two controllable variables. This study conducts an online survey of those in Taiwan who have experience in online 
shopping and goods pick-up at convenience stores, in order to investigate how customer satisfaction and switching cost influence customer loyalty, we study the pick-up point loyalty intentions of existing customers for two convenience stores.

The purpose of this study was to explore the relationships between service quality, switching cost and loyalty using a nonlinear dynamic modeling approach. In accordance with the catastrophe theory, results were analyzed using the program GEMCAT II. The contributions of our work are as follows.

1. In the cusp catastrophe model, "Reselection electronic map" is the main index of the splitting factor. "Relative service quality concerning the electronic map" is the main index of the normal factor.

2. These characteristics including bimodality, hysteresis and catastrophe are all present in our research data.

3. With high switching cost, whether a person is satisfied or dissatisfied, it is very difficult to change to the opposite state. On the other hand, if a change is effected, our model would predict that it will persist with the same degree of "inertia" which maintains the original state.

4. According to the previous findings of loyalty within the application of the cusp catastrophe theory, the cusp catastrophe model is an appropriate model for understanding the process of loyalty.

We hope that we made clear that the catastrophe approach to discontinuous behavior has fruitful implications. Catastrophe theory concerns qualitative behavior of continuous variables. It suggests a complex relation between continuous and categorical variables, which falls outside the scope of standard categorical models and data-analytical methods. It is suggested that other researchers could consider the cusp catastrophe theory and other nonlinear techniques, especially when standard approaches do not adequately capture the underlying dynamics.

\section{References}

1. D.J. Wales, A microscopic basis for the global appearance of energy landscapes, Science, Vol. 293 (2001), pp. 2067-2070.

2. S. Qin, J.J. Jiao, S. Wang and H. Long, A nonlinear catastrophe model of instability of planar-slip slop and chaotic dynamical mechanisms of its evolutionary process, International Journal of Solids and Structures, Vol 38 (2001), pp. 8093-8109.

3. D. Aerts, M. Czachor, L. Gabora, M. Kuna, A. Posiewnil, J. Pykacz and M. Syty, Quantum morphogenesis: A variation on Thom's catastrophe theory, Physical Review E, Vol. 67 (2003), pp. 1-13.

4. X.A. Lignous, G.A.R. Parke, J.E. Harding and A.N. Kounadis, A comprehensive catastrophe theory for non-linear buckling of simple system exhibiting fold and cusp catastrophe, International Journal for Numerical Methods in Engineering, Vol. 54 (2002), pp. 175-193.

5. T.Y. Kenneth and S.O. Cheung, A catastrophe model of construction conflict behavior, Building and Environment, Vol.41 (2006), pp. 438-447.

6. I.N. Stewart and P.L. Peregoy, Catastrophe theory modeling in psychology, Psychological Bulletin, Vol. 94 (1982), pp. 336-362.

7. T.A. Oliva, R. L. Oliver and I.C. MacMillian, A Catastrophe Model for Developing Service Satisfaction Strategies, Journal of Marketing, Vol. 56 (1992), pp. 83-95.

8. M. Smith, R.A. Lancioni and T.A. Oliva, The effects of management inertia on the supply chain performance of produce-to-stock firms, Industrial Marketing Management, Vol. 24 (2005), pp.614-628. 
9. W. Dou and S. Ghose, A dynamic nonlinear model of online retail competition using cusp catastrophe theory, Journal of Business Research, Vol. 59 (2006), pp.838-848.

10. G.G. Haveman and T.A. Oliva, Organization design, inertia, and the dynamics of competitive response, Organization Science, Vol. 4 (1993), pp. 181-208.

11. G.K. Kalph and T.A. Oliva, Multivariate Catastrophe Model Estimation: Method and Application, Academy of Management Journal, Vol. 37 (1994), pp. 206-221.

12. T.A. Olvia, W.S. Desarbo, D.L. Day and K. Jedidi, GEMCAT: A GEneral Multivariate methodology for estimating CATastrophe models, Behavior Science, Vol.32 (1987), pp. 121-137.

13. L. Lange, S. McDade and T.A. Oliva, Technological Choice and Network Externalities: A Catastrophe Model Analysis of Firm Software Adoption for Competing Operating Systems, Structural Change and Economic Dynamics, Vol. 12 (2001), pp. 9-57.

14. C.M. Feng and Y.K. Huang, The Choice Behavior Analysis of the Pick-up Point for the E-commerce Retailing Delivery, Journal of the Eastern Asia Society for Transportation Studies, Vol. 6 (2005), pp. $2778-2793$. 